\title{
Cognition, psychosis risk and metabolic measures in two adolescent birth cohorts
}

Hugh Ramsay, Jennifer H Barnett, Graham K Murray, Jouko Miettunen, Pirjo Mäki, Marjo-Riitta Järvelin, George Davey Smith, Mika Ala-Korpela, Juha Veijola

Hugh Ramsay: Department of Psychiatry, Research Unit of Clinical Neuroscience, University of Oulu, Oulu, Finland; Department of Psychiatry, Royal College of Surgeons in Ireland, Dublin, Ireland; St. Michael's House, Dublin, Ireland; +353 86 3859921; drhughramsay@gmail.com

Jennifer H Barnett: Department of Psychiatry, University of Cambridge, Cambridge, United Kingdom; Cambridge Cognition Ltd, Cambridge, UK; +44 1223 337733; jhb32@cam.ac.uk

Graham K Murray: Department of Psychiatry, University of Cambridge, Cambridge, United Kingdom; +44 1223 337733; gm285@cam.ac.uk

Pirjo Mäki: Department of Psychiatry, Research Unit of Clinical Neuroscience, University of Oulu, Oulu, Finland; Department of Psychiatry, University Hospital of Oulu, Oulu, Finland; Department of Psychiatry, Länsi-Pohja healthcare district, Finland, Department of Psychiatry, the Middle Ostrobothnia Central Hospital, Kiuru, Finland; Mental health services, Joint Municipal Authority of Wellbeing in Raahe District, Finland; Mental health services, Basic Health Care District of Kallio, Finland; Department of Psychiatry, Kainuu Central Hospital, Kainuu Social and Healthcare District, Finland; +358 294 480000; pirjo.maki@oulu.fi 
Jouko Miettunen: Center for Life Course Health Research, Faculty of Medicine, University of Oulu, Oulu, Finland; +358 294 480000; jouko.miettunen@oulu.fi Marjo-Riitta Järvelin: Department of Epidemiology and Biostatistics, MRC-PHE Centre for Environment and Health, Imperial College London, London, W2 1PG, United Kingdom; Center for Life Course Health Research, Faculty of Medicine, University of Oulu, Finland; Biocenter Oulu, University of Oulu, Aapistie 5, 90220 Oulu, Finland; Unit of Primary Health Care, Oulu University Hospital, OYS, Kajaanintie 50, 90220, Oulu, Finland; Department of Life Sciences, College of Health and Life Sciences, Brunel University London, Kinston Lane, Uxbridge, Middlesex UB8 3PH, United Kingdom; m.jarvelin@imperial.ac.uk

George Davey Smith: Medical Research Council Integrative Epidemiology Unit (IEU), Bristol Medical School, University of Bristol, Bristol, United Kingdom; KZ.Davey-Smith@bristol.ac.uk

Mika Ala-Korpela: Systems Epidemiology, Baker Heart and Diabetes Institute, Melbourne, Victoria, Australia; Computational Medicine, Faculty of Medicine, University of Oulu and Biocenter Oulu, Oulu, Finland; NMR Metabolomics Laboratory, School of Pharmacy, University of Eastern Finland, Kuopio, Finland; Population Health Science, Bristol Medical School, University of Bristol, Bristol, United Kingdom; Medical Research Council Integrative Epidemiology Unit at the University of Bristol, Bristol, United Kingdom; Department of Epidemiology and Preventive Medicine, School of Public Health and Preventive Medicine, Faculty of Medicine, Nursing and Health Sciences, The Alfred Hospital, Monash University, Melbourne, Victoria, Australia; mika.ala-korpela@baker.edu.au Juha Veijola: Medical Research Center Oulu, University Hospital of Oulu and University of Oulu, Finland; Department of Psychiatry, Research Unit of Clinical 
Neuroscience, University of Oulu, Oulu, Finland; Department of Psychiatry, University Hospital of Oulu, Oulu, Finland; +358 04 5237413;

juha.veijola@oulu.fi

Corresponding author = Hugh Ramsay, St Michael's House, Ballymun Road, Dublin 9, Ireland

Word count: 5,318 


\begin{abstract}
Background: Psychoses, especially schizophrenia, are often preceded by cognitive deficits and psychosis risk states. Altered metabolic profiles have been found in schizophrenia. However, the associations between metabolic profiles and poorer cognitive performance and psychosis risk in the population remain to be determined.
\end{abstract}

Methods: Detailed molecular profiles were measured for up to 8,976 individuals from two general population-based prospective birth cohorts: the Northern Finland Birth Cohort 1986 (NFBC 1986) and the Avon Longitudinal Study of Parents and Children (ALSPAC). A high-throughput NMR spectroscopy platform was used to quantify 70 metabolic measures at age $15-16$ years in the NFBC 1986 and at ages 15 and 17 years in ALSPAC. Psychosis risk was assessed using the PROD-screen questionnaire at age 15-16 years in the NFBC 1986 or the PLIKS assessment at age 17 in ALSPAC. Cognitive measures included academic performance at 16 years in both cohorts and general intelligence and executive function in ALSPAC. Logistic regression measured cross-sectional and longitudinal associations between metabolic measures and psychosis risk and cognitive performance, controlling for important covariates.

Results: Seven metabolic measures, primarily fatty acid measures, showed cross-sectional associations with general cognitive performance, four across both cohorts (LDL diameter, MUFA ratio, omega-3 ratio and DHA ratio), even after controlling for important mental and physical health covariates. Psychosis risk showed minimal metabolic associations. 
Conclusions: Fatty acid ratios may be important in marking risk for cognitive deficits in adolescence. Further research is needed to clarify whether these biomarkers could be causal and thereby possible targets for intervention.

Keywords: psychosis risk, cognition, metabolomics, lipoprotein lipids, fatty acids, NFBC 1986, ALSPAC 


\section{Introduction}

Brain structure and function develop rapidly during adolescence. Problems with this development may be evident in cognitive performance problems or in psychiatric symptoms in response to increasing challenges in the adolescent environment. Psychoses, especially schizophrenia and psychotic disorders are conceptualised as neurodevelopmental disorders (Murray \& Lewis 1988), the result of a series of insults in healthy brain development. In this neurodevelopmental process, the adolescent period plays an important role (Rapoport et al. 2005; Paus et al. 2008). Risk for psychosis is increasingly being measured at this age, both in clinical samples (Fusar-Poli et al. 2013) and at a general population level (Kelleher et al. 2012). The static and dynamic cognitive deficits that are known to precede schizophrenia, such as deficits in general intelligence and specific cognitive domains, have also increasingly been considered (Reichenberg et al. 2010), both as markers of risk for later illness and as independent predictors of functioning in young adults with mental disorders (Fett et al. 2011; McIntyre et al. 2013; Holshausen et al. 2014) and at risk states for mental disorders (Carrión et al. 2013). Better understanding of the biological signature of brain development and of relative problems in brain development 
has the potential to improve public mental health and individual functional outcomes.

Metabolomics approaches utilising nuclear magnetic resonance (NMR) spectroscopy have shown increasing promise in characterising the molecular signature of body fluids for disease risk (Ala-Korpela 2007a, 2007b). NMR spectroscopy has particularly suited study of the molecular signature of disease and event risk in larger epidemiological samples. The approach has uncovered metabolic associations with: disease risk states, such as obesity (Würtz et al. 2014) and insulin resistance (Würtz et al. 2012); illnesses and illness events, such as diabetes (Wang et al. 2015), cardiovascular events (Würtz et al. 2015), and all-cause mortality (Fischer et al. 2014); and health-related behaviours, such as alcohol consumption (Würtz et al. 2016) and physical activity (Kujala et al. 2013).

In the study of brain development and disorders, NMR spectroscopy approaches have identified patterns regarding schizophrenia (Holmes et al. 2006) and mild cognitive impairment in older people (Tukiainen et al. 2008), and have suggested possible molecular associations with cognitive reaction times in young people with schizophrenia (Scoriels et al. 2015). Mass spectrometry metabolomics 
approaches have also shown promise in mapping molecular features of schizophrenia (Orešič et al. 2011; He et al. 2012; Yang et al. 2013) and of cognitive impairment in older people (Trushina et al. 2013; Mapstone et al. 2014).

Difficulties in adolescent cognitive performance and psychosis risk are associated with negative outcomes in adulthood. To our knowledge, metabolomics approaches have not been utilised to examine these adolescent characteristics at a general population level. Characterisation of the metabolic measures associated with relatively poorer cognition and psychosis risk in adolescence has the potential to improve understanding of their development. The present study examines if there are cross-sectional and longitudinal associations between a panel of metabolic measures and broad cognitive ability and psychosis risk in late adolescence. 


\section{Methods}

Study populations

Serum metabolic profiles were quantified from two independent general population-based birth cohorts: the Northern Finland Birth Cohort 1986 (NFBC 1986) and the Avon Longitudinal Study of Parents and Children (ALSPAC) from the United Kingdom (UK).

The NFBC 1986 is a longitudinal birth cohort, covering 99\% of births in the two northernmost provinces of Finland, Oulu and Lapland, who had an expected delivery date between July $1^{\text {st }} 1985$ and June $30^{\text {th }} 1986$, details of which have been described elsewhere (Järvelin et al. 1993; http://kelo.oulu.fi/NFBC/). In brief, the cohort consisted of 9,432 live-born children, of whom 6,985 (76\%) completed questionnaires (Miettunen et al. 2008; Taanila et al. 2009; Koivukangas et al. 2010; Mäki et al. 2014; Metcalf et al. 2017) and 5,606 (59\%) provided serum samples for NMR metabolomics analysis at age 15-16 years. The study received ethics approval from the ethics committees of the Northern Ostrobothnia Hospital District and the University of Oulu and all participants and their parents provided written informed consent.

ALSPAC is a longitudinal birth cohort, consisting of $72 \%$ of eligible expectant mothers in Bristol, UK, and surrounding areas, who had an expected delivery date between April $1^{\text {st }} 1991$ and December 31 ${ }^{\text {st }} 1992$, details of which have been described elsewhere (Golding et al. 2001; Boyd et al. 2013; 
http://www.bristol.ac.uk/alspac/). In brief, this consisted of 14,062 live-born children. Of these, 9,985 were invited to follow-up at age 15 years and 5,253 attended (and 3,366 provided plasma samples for NMR-based metabolomics). A further 10,101 were invited to follow-up at age 17 years (including 782 not in the original sample), of whom 5,217 attended (and 3,176 provided plasma samples for NMR-based metabolomics). Please note that the study website contains details of all the data that is available through a fully searchable data dictionary (http://www.bris.ac.uk/alspac/researchers/data-access/datadictionary/). Ethical approval for the study was obtained from the ALSPAC Law and Ethics Committee and the Local Research Ethics Committees, and all participants and their parents provided written informed consent.

\section{Molecular profiling}

NMR spectroscopy allows for the application of metabolomics approaches in large-scale epidemiological samples (Soininen et al. 2015) and has been extensively used in multiple large-scale epidemiological studies (Mahendran et al. 2013; Auro et al. 2014; Fischer et al. 2014; Würtz et al. 2015; Würtz et al. 2016). The precise methodology is described elsewhere (Soininen et al. 2009, 2015). In brief, the NFBC 1986 participants provided serum samples at age 16 years, while ALSPAC participants provided EDTA plasma samples at ages 15 and 17 years and these were stored at -80C for subsequent biomarker profiling. A high-throughput NMR spectroscopy metabolomics platform was used for the quantification of 70 metabolic measures (Soininen et al. 2015), representing a broad molecular signature of systemic metabolism, including lipoprotein lipids 
and subclasses, lipoprotein size, fatty acids, ratios of fatty acid subclasses to total fatty acids, amino acids, gluconeogenesis- and glycolysis-related metabolites, ketone bodies and others. All measurements were performed at the NMR Metabolomics Laboratory at the University of Eastern Finland, Kuopio, Finland. Uniform sample preparation protocols and NMR instrumentation and parameter were used. Details have been described elsewhere (Soininen et al. 2009; Inouye et al. 2010). The NFBC 1986 and ALSPAC samples were measured separately using identically calibrated instrumentation, though it is notable that NMR-based metabolic quantifications are stable over long periods (Kettunen et al. 2016). Standardised protocols were used to minimise any issues around sample transport and sample age.

\section{Academic performance}

Our outcomes of interest were cognitive measures and psychosis risk across the two samples. Our main cognitive measure was academic performance, measured in both samples at age 16 years. In the NFBC 1986 information on academic performance was provided by the National Application Register for Upper Secondary Education, Finland, based on nationally comparable grades of the final assessment of basic education. They describe the level of performance in relation to the objectives of basic education at the end of grade 9 (age 16). In ALSPAC the data on academic performance provided by the United Kingdom Department of Education, where performance was measured using the overall points score derived from the General Certificate in Secondary Education (GCSE) or equivalent exam. As we were interested in predictors of poorer performance, 
in both samples the results were categorised to compare those with performance $>1$ standard deviation below the mean with the remaining group with better performance.

\section{Cognition}

Participants in the ALSPAC study performed additional cognitive tests at age 15 and 17 years. The Wechsler Abbreviated Scale of Intelligence (WASI) was performed at age 15 years, at the same time as the measurement of metabolic measures. The N-back and Probability Reversal (PR) tests were performed at age 17 years, 2 years after measurement of metabolic measures. The WASI is a valid brief measure of general intelligence in children as well as adults (Saklofske et al. 2000; Abu-Hilal et al. 2011). The N-back and Probability Reversal each measure components of executive function. The N-back task is a measure of working memory (Miller et al. 2009). We utilised target identification accuracy on the 2back task for this test. Probability Reversal is a test of ability to adapt to specific contingencies. We utilised latency in stage 2 for this test, which was logtransformed for normality.

Psychosis risk

Psychosis risk in adolescence were measured at age 16 years using the PRODscreen questionnaire (Heinimaa et al. 2003) in the NFBC 1986 (Hurtig et al. 2011; Therman et al. 2011; Mäki et al. 2014). This screening questionnaire includes twenty-one questions measuring lower level psychosis risk. Full- 
information item factor analysis has identified a three-dimensional (positive, negative and general) factor structure to the PROD-screen (Therman et al. 2011). Based on these dimensions, we classified individuals as having psychosis risk where they endorsed six or more out of a total of eleven positive items (412/6,041,6\%). This cut-off was utilised based on meta-analysis suggesting that the prevalence of psychotic experiences at age 16 is about 7.5\% (Kelleher et al. 2012).

Psychosis risk among ALSPAC participants was measured using clinical interview for psychotic-like experiences ("PLIKS") clinical interview at age 17 years, which was conducted by trained psychology graduates. Three main domains of positive psychotic symptoms were elicited: hallucinations, delusions, and thought interference. Interviewers rated psychosis risk as absent, suspected or definitely psychotic. We classified individuals as having psychosis risk where they showed one or more definite symptoms on interview (Zammit et al. 2009). Based on these criteria, we classified 232/4,480 (5\%) as having psychosis risk in this sample.

\section{Confounding variables}

We considered three main potential confounders to any association between the metabolic measures and cognitive performance/psychosis risk: sex, socioeconomic status and body mass index. Sex is associated with academic performance and also with levels of various metabolic measures (Serbin et al. 1990; Ishikawa et al. 2014). Socioeconomic status is associated with academic 
performance and with lifestyle factors (e.g. diet) likely to influence metabolic measures (Coe et al. 2013). The socioeconomic status of adolescents in the NFBC 1986 was controlled for using the variable "maternal education". At birth, mothers were asked about their own level of education attainment and this was classified into four groups: (1) up to 8 years of primary education; (2) 9-10 years of primary education; (3) vocational school or college for at least 6 months and; (4) commenced university education. Socioeconomic status was controlled for using the social class of the mother based on occupation in the ALSPAC sample. This included 6 groups: professional, managerial and technical, skilled nonmanual, skilled manual, partly skilled, and unskilled. Both socioeconomic variables were treated as linear for the purposes of analyses. Body mass index (BMI) was calculated from height and weight, which were measured during clinical examination in adolescence for both samples. The standard formula for BMI was used (weight(kg)/height $\left.(\mathrm{m})^{2}\right)$.

In addition to controlling for these factors across both cohorts, it was possible to control for additional mental health factors (anxiety and depression, psychosis risk) and physical health factors (visits to a doctor in the past six months, physical exercise, healthy diet) in the NFBC sample. Anxiety and depression were measured at age 16 years using sixteen questions from the Youth Self Report (Song et al. 1994). Psychosis risk was assessed as outlined above, using the PROD-screen (Heinimaa et al. 2003). Adolescents were asked at age 16 years about their physical health, physical exercise and diet. Number of visits to the doctor in the previous 6 months was used as a proxy for physical health, comparing those with 2 or more visits with those with $0-1$ visits. Those who 
described lower levels of physical activity were compared with those with higher levels of physical activity, comparing 0-1 hour per week of brisk physical activity with $>1$ hour per week, excluding physical activity during school hours (Koponen et al. 2008). Diet was considered healthy or unhealthy, depending on the amount of fruits, vegetables and berries used (with healthy considered as any of these on three or more days per week) (Koponen et al. 2008).

\section{Statistics}

Cognitive measures were transformed into binary outcomes for ease of interpretation, comparing individuals more than 1 standard deviation (SD) below the mean scores to the remainder of the sample. Psychosis risk status was a binary outcome, as described above. Metabolic measures with skewed distribution were normalised by log-transformation prior to analyses and all metabolic measures were z-transformed to examine the effect of each 1 standard deviation increase on the odds for the cognitive outcome. The metabolic measures were therefore transformed into ordinal measures for interpretation but they were treated as continuous for the purpose of analysis. Due to the correlated nature of the data, principal component analysis was used to evaluate the appropriate number of independent tests to correct for multiple testing. In this case, $90 \%$ of the variance in the 70 measures was explained by at most 12 principal components in each cohort. Multiple testing correction therefore accounted for 12 independent tests using the Bonferroni method, resulting in a corrected P-value of $<0.004$. 
Our primary hypotheses were that cognitive difficulties and psychosis risk status (the outcomes of interest) were associated with differences in metabolic measures (the main exposure of interest), both cross-sectionally and longitudinally. The temporal relationship between exposures and outcomes and the cross-sectional and longitudinal associations under review are presented in figure 1. For the cross-sectional analyses, a logistic regression model was fitted for each outcome measure (academic performance at age 16 years in the NFBC 1986, psychosis risk at age 16 in the NFBC 1986, WASI performance at age 15 for ALSPAC, executive skills performance at age 17 years for ALSPAC, and psychosis risk at age 17 years for ALSPAC), using metabolic measures as the explanatory variable. Individuals with higher cognitive performance or lower psychosis risk were used as the reference group, so the odds ratios calculated reflect the association between increasing standard deviations of metabolic measures and poorer cognitive performance/higher psychosis risk. The two cohorts were analysed separately. In all analyses we initially adjusted for sex (model 1). Where an association was observed at the required P-value, we sequentially adjusted for socioeconomic status (model 2) and body mass index (model 3).

Additional longitudinal analyses were performed with ALSPAC (see figure 1). In this case, cognitive performance and psychosis risk were also the outcomes of interest and metabolic measures were the explanatory variables of interest. Logistic regression models were fitted for each outcome (academic performance at age 16 years, executive function at age 17 years, psychosis risk at age 17 years) with metabolic measures taken at age 15 years as the explanatory variables of interest. In these analyses we initially adjusted for sex (model 1) 
and, where an association was observed at the required P-value, we then adjusted sequentially for socioeconomic status (model 2) and body mass index (model 3).

Secondary hypotheses were tested in the cohorts separately. Our first secondary hypothesis was that metabolic associations with cognitive performance were not explained by anxiety and depression but were influenced by psychosis risk status. In order to test this, we compared model 3 above to a model controlling for depression and anxiety (model 4) in the NFBC 1986. We then compared this model 4 (or model 3 in the case of the ALSPAC) with a further model, controlling for psychosis risk (model 5). These models controlling for psychosis risk were then compared with a model considering interaction between psychosis risk and the metabolic measures. Our second secondary hypothesis was that metabolic associations with cognitive performance were not explained by physical health factors. This could only be tested in the NFBC 1986 sample, by comparing model 3 to a model controlling for doctors visits in the previous six months, physical exercise and healthy diet (model 6). 


\section{Results}

The characteristics of participants in the two cohorts are given in Table 1. Higher academic performance in both samples was associated with female sex, with having mothers who were more educated or from a higher social class and with lower mean body mass index (more markedly in ALSPAC). In the NFBC 1986 sample, higher academic performance was associated with higher score on anxiety and depression items $(\mathrm{P}<0.001)$, higher rates of more physical activity $(\mathrm{P}<0.001)$ and higher rates of healthy $\operatorname{diet}(\mathrm{P}<0.001)$. In the ALSPAC sample, higher academic performance was associated with lower rates of psychosis risk $(\mathrm{P}<0.001)$

Cross-sectional associations between metabolic measures and cognitive performance and psychosis risk

A range of apolipoprotein and fatty acid-related metabolic measures showed cross-sectional associations with broad cognitive performance (see figure 2). In the NFBC 1986 sample, 18 metabolic measures were associated with academic performance at age 16. These included lipids in lipoproteins, lipoprotein diameters, triglyceride measures and, particularly frequently, fatty acid ratios. Among these, LDL diameter, MUFA level, ratios of fatty acid subclasses (MUFA, PUFA, omega-6, omega-3, DHA, \% unsaturation) to total fatty acids, citrate and creatinine remained associated with academic performance even after controlling for maternal education and body mass index (see table 2). In ALSPAC, nine metabolic measures were associated with general intelligence scores at age 
15 years. Among these, lipids in small HDL, LDL diameter, fatty acid levels (omega-3, DHA), and ratios of fatty acid subclasses (MUFA, omega-3, DHA) to total fatty acids were associated at $\mathrm{P}<0.004$ after controlling for maternal social class and body mass index.

There were no cross-sectional associations at $\mathrm{P}<0.004$ between any of the metabolic measures at age 17 years and Probability Reversal at age 17 years (see supplementary figure 1). In the case of N-back accuracy, increasing standard deviations of the two DHA measures were associated with better performance. DHA level at age 17 years was associated in model 1 (OR=0.81, 95\% CI: 0.700.93, $\mathrm{P}=0.004)$, borderline associated in model 2 (OR=0.79, 95\% CI: 0.66-0.93, $\mathrm{P}=0.006)$ and associated in model $3(\mathrm{OR}=0.77,95 \% \mathrm{CI}: 0.65-0.92, \mathrm{P}=0.003)$. Ratio of DHA to total fatty acids at age 17 years was associated with N-back accuracy in model 1 (OR=0.78, 95\% CI: 0.68-0.90, P=0.001), model 2 (OR=0.76, 95\% CI: $0.64-$ 0.90, $\mathrm{P}=0.001)$, and model $3(\mathrm{OR}=0.76,95 \% \mathrm{CI}: 0.64-0.90, \mathrm{P}=0.001)$.

There was minimal evidence against the null hypothesis for cross-sectional associations between fatty acid levels and psychosis risk in the NFBC 1986 sample, with none showing associations at $\mathrm{P}<0.004$ (see supplementary figure 2). In the ALSPAC sample, there were weak cross-sectional associations between DHA levels and psychosis risk, though these did not reach our P-value threshold (see supplementary figure 2). One metabolic measure, acetate, was associated with lower risk for psychosis (OR=0.37, 95\% CI: 0.16-0.57, $\mathrm{P}<0.001)$. The evidence for this association against the null hypothesis was weaker after 
controlling for maternal social class $(\mathrm{OR}=0.38, \mathrm{P}=0.003)$ and $\mathrm{BMI}(\mathrm{OR}=0.42$, $\mathrm{P}=0.008)$.

Longitudinal associations between metabolic measures and cognitive performance and psychosis risk

The longitudinal associations between metabolic measures and cognitive performance are presented in figure 3. In brief, there were various associations between metabolic measures associated with apolipoproteins and fatty acids and cognitive function longitudinally. Specifically, nine measures at age 15 years were associated with academic performance at age 16 years (lipids in large HDL, LDL diameter, HDL diameter, MUFA, DHA, ratios of fatty acid subgroups to total fatty acids) (see table 3). Among these, four remained associated after controlling for maternal social class and body mass index: higher LDL diameter, MUFA level, ratios of MUFA and DHA to total fatty acids. Five fatty acid measures at age 15 years were associated with Probability Reversal latency at age 17 years (PUFA, omega-6, linoleic acid, omega-3 and DHA) at $\mathrm{P}<0.004$ and these associations were broadly similar after controlling for maternal social class and body mass index.

No metabolic measures at age 15 years were associated with N-back accuracy at 17 years or psychosis risk at 17 years (see supplementary figure 3).

The observed cross-sectional and longitudinal associations are presented together in table 4. 
Metabolic measures and cognitive performance in the context of mental and physical health factors

Cross-sectional associations between metabolic measures and cognitive performance, which were significant in both samples, were further tested by controlling for mental health factors and physical health factors. Results of these analyses are presented in table 5. The metabolic measures-cognitive associations were not attenuated in the NFBC 1986 sample after controlling for anxiety and depression at age 16 years. The associations were also not attenuated after controlling for psychosis risk, in either the NFBC 1986 or the ALSPAC. There were no interactions between the metabolic measures and psychosis risk in predicting cross-sectional cognitive outcomes for the NFBC 1986 or the ALSPAC.

There was no attenuation in the association between metabolic measures and academic performance after controlling for physical health factors (doctors visits in the previous 6 months, exercise level and healthy diet) in the NFBC 1986 (see table 5 to compare models). 


\section{Discussion}

In this study across two general population-based cohorts, we have characterised the associations between metabolic measures and broad brain function in late adolescence. The main findings were that fatty acid metabolic measures and, to a lesser extent, apolipoprotein measures, were associated with adolescent brain function. These findings were stronger and more consistent in cross-sectional analyses and where academic scores and general intelligence were the outcomes. These findings did not appear to be related to differences in mental health factors or physical health factors between groups. Findings in the case of executive function were more nuanced, with associations between fatty acid measures at age 15 years and one of our two tests of executive function (PR latency) at age 17 years, and weaker cross-sectional associations also observed for omega-3 at age 15 years. Findings for psychosis risk were fewer and weaker.

Metabolic measures and general intelligence

While two lipoprotein metabolic measures (lipids in small HDL, LDL diameter) were associated with general intelligence, a range of fatty acid levels and ratios were associated on both cross-sectional and longitudinal analyses. The presence of cross-sectional and longitudinal associations across two cohorts adds weight to these results. The observed associations for general intelligence are also meaningful in their strength. Each standard deviation increase in these fatty acid measures showed reduced odds of between 0.62 and 0.87 or increased odds of between 1.15 and 1.50 of performing more than 1 standard deviation from the 
mean (poorest performing $15.8 \%$ of sample). While these associations are not large, they are meaningful.

There are a number of potential explanations for the findings. Firstly, there may be lifestyle factors, associated with academic performance and fatty acid ratios that are playing a causal role. Placing these results in the context of other metabolomics work provides potential clues in this area. The pattern of fatty acids observed here has been noted in some other studies, particularly with regard to physical activity (Kujala et al. 2013) and cardiovascular events (Würtz et al. 2015), while different patterns have been observed for other outcomes (e.g. for alcohol consumption (Würtz et al. 2016)). It was therefore possible that observed associations between metabolic measures and cognition reflected a generally healthy metabolic profile associated with cognitive performance or the effects of physical activity on cognitive performance. This study suggests this explanation is unlikely. Controlling for three significant physical health factors (doctor visits, physical exercise and diet) did not significantly impact on the associations between the four metabolic factors most consistently associated with cognitive performance. In addition, controlling for significant mental health factors that impact on cognition, anxiety and depression and psychosis risk, did not impact on the observed associations.

Secondly, the metabolic measures may be playing a causal role in terms of general cognitive ability. However, the results observed regarding omega-3 ratios provide very limited evidence in support of Bradford Hill's criteria around causality (Bradford Hill 1965). These general guidelines suggest that strength, 
consistency and specificity of associations support causality, along with temporality, biological gradient, plausibility, coherence, experiment and analogy. Specifically, we have observed a moderate strength association, consistent findings between studies and limited temporal associations. One plausible mechanism is that an effect on general cognitive performance could be mediated through previously identified effects of omega-3 fatty acids on attention (Bos et al. 2015), which could enhance general cognitive development. Thirdly, higher academic performance (or a factor for which academic performance is a proxy) may precede the metabolic findings. Further longitudinal work would be needed to clarify this.

Fatty acid ratios showed more consistent associations with general cognitive performance than individual fatty acid levels in this study. This should be considered in light of the correlation between absolute fatty acid levels and lipoprotein lipids, particularly triglycerides. Fatty acid ratios are therefore more reflective of the individual role of these fatty acids than fatty acid levels themselves. The general pattern of associations was that higher ratios of polyunsaturated fatty acids were associated with better performance while higher ratios of monounsaturated fatty acids were associated with poorer performance. Long chain PUFAs are important in cell membrane structure and function. This role may be particularly important in adolescence, a time when psychiatric neurodevelopmental disorders associated with cognitive deficits (such as schizophrenia) begin to emerge. Higher ratios of MUFA and lower ratios of PUFA and its sub-classes may therefore be beneficial, or reflect a beneficial environment, in terms of adolescent neuronal membrane function. 
Metabolic markers and executive function

The findings with regard to executive function are less consistent than those for general intelligence. For one test (N-back), DHA levels and ratios showed crosssectional associations but there were no longitudinal associations. For the other test (Probability Reversal), there were no cross-sectional associations but fatty acid levels at age 15 years (PUFAs, omega-6, linoleic acid and DHA) were associated with performance at 17 years. Once again, fatty acid measures, particularly DHA, showed the most noteworthy effects. This may suggest that PUFA levels in mid-adolescence are particularly important in executive function. It is interesting that the cross-sectional findings were present in the N-back task, which primarily assesses working memory, while the longitudinal findings were present for the Probability Reversal task, which assesses learning following a rule change. This aspect of executive function is evolving rapidly during adolescence (Blakemore \& Choudhury 2006) and this may partly explain the differences in longitudinal and cross-sectional findings between the tests. However, given the variability in the findings, further replication is warranted.

Metabolic markers and psychosis risk

While we observed one cross-sectional association between acetate and psychosis risk in ALSPAC, this finding was not evident in the NFBC sample or in ALSPAC longitudinally. Other findings were weak and not consistent between samples or between cross-sectional and longitudinal analyses. One possible 
reason for differences in findings between the NFBC 1986 and ALSPAC is that the measures used were quite different. The PROD-screen is a screening questionnaire and has limited evidence of either sensitivity or specificity at the cutoff used here (around 6\% of the NFBC 1986 sample). It is therefore likely that among those who scored positive, there were many without psychosis risk as well as those with them. The PLIKS interview was more specific for psychosis risk but the outcome was therefore rarer (only $5 \%$ of the ALSPAC group). Ideally studies would utilise the same measures to allow for potential replication.

Psychosis risk did not appear to play a major role in the cognitive findings. Controlling for psychosis risk did not impact on cognitive findings. This may be due to the relatively low numbers with psychosis risk (5-6\% of the samples). However, the findings suggest that, at a population level at least, metabolic measures do not influence cognition differentially according to psychosis risk. Further studies with enriched psychosis risk samples may be useful to consider this further.

\section{Future research implications}

We have observed cross-sectional and longitudinal associations between metabolic markers and cognition. Future research should examine other specific cognitive domains outside of executive function. Future studies could add to these findings by further exploring for evidence in support of causality, for example by considering potential biological mechanisms through experimental animal studies. In addition, consideration of the role of other covariates, such as 
exercise, would be helpful, even if this is on the same causal pathway to the outcome.

\section{Limitations and advantages}

A number of limitations of this study must be acknowledged. Firstly, much of the data was cross-sectional, making it difficult to more closely examine the directions of relationships. However, longitudinal associations between fatty acid metabolic measures and academic performance and executive performance in the ALSPAC study add considerably to the cross-sectional findings. Secondly, we could control for only a limited number of potential confounders in the ALSPAC group. However, those chosen (maternal education, body mass index) at least partly address a wide variety of potential confounders such as diet and exercise levels. Furthermore, controlling for physical health, diet and exercise did not impact on observed associations in the case of the NFBC 1986 sample. Thirdly, the main outcome of interest, academic performance, has limitations. In addition to reflecting a variety of cognitive processes (Rohde \& Thompson 2007), academic performance reflects self-discipline (Duckworth \& Seligman 2005), sex and home cultural factors, including socio-economic context. This could also be regarded as a strength, as academic achievement reflects a variety of personality and cognitive processes that are important for success into adulthood. In addition, this data was augmented with more specific measures of executive function, a key cognitive skill that advances rapidly during late adolescence. Fourthly, the psychosis risk outcomes had limitations, particularly in the case of the PROD-screen items, which has limited validity in identifying psychosis risk. 
Fifthly, the use of body mass index in adolescence has limitations, as it is likely to fluctuate in adolescence in response to growth spurts. However, the use of other physical measures in the NFBC 1986 sample partly addresses this limitation. Finally, the conversion of measurements, including cognitive performance, from continuous into categorical will result in some loss of information. However, this loss of information is justified by the measures being more meaningful and easier to interpret.

This study also has a number of important strengths. The data is derived from two large general population-based birth cohorts. Despite this, the findings were similar across the studies. Furthermore, the two samples come from different genetic contexts and different social contexts, including with regard to diet, which reduces the chances of uncontrolled confounding. As mentioned, information was both cross-sectional and longitudinal. We were also fortunate to have data on a large number of metabolic measures and sufficient participants to truly examine associations across two samples. Finally, we were fortunate that for the NFBC 1986 sample, we had rich data on physical and mental health factors that may have impacted on the results.

\section{Conclusion}

In conclusion, we have identified differences in fatty acid ratios associated with academic and cognitive performance in adolescence. These were strongest for general cognitive ability and were not explained by mental or physical health factors. Further analysis of these fatty acid ratios may further our understanding 
of cognitive development at this key age, particularly in groups that are especially vulnerable to psychiatric co-morbidity and longer-term functional deficits.

\section{Acknowledgements:}

We are extremely grateful to all the families who took part in these studies and the whole NFBC 1986 and ALSPAC teams, which includes interviewers, computer and laboratory technicians, clerical workers, research scientists, volunteers, managers, receptionists and nurses. We thank professor Anna-Liisa Hartikainen (who launched the NFBC 1986), the NFBC project center and the University of Oulu. The UK Medical Research Council and Wellcome (Grant ref: 102215/2/13/2) and the University of Bristol provide core support for ALSPAC, while ALSPAC metabolomics data generation was funded by the UK Medical Research Council (MRC - MC_UC_12013/1). This research was also supported by funding from the EU (QLG1-CT-2000-01643 (EUROBLCS) Grant no. E51560), NorFA (Grant no. 731, 20056, 30167), the NIHH (USA) 2000 (G DF682 Grant no. 50945), and the researchers' own funding. JM was supported by the Academy of Finland (Grant no. 268336). MAK was supported by the Sigrid Juselius Foundation, Finland. MAK and GDS work in a Unit that is supported by the University of Bristol and UK Medical Research Council (MC_UU_12013/1). GDS works in the Medical Research Council Integrative Epidemiology Unit at the University of Bristol (MC_UU_00011/1). This publication is the work of the authors and they will serve as guarantors for the contents of the paper. 


\section{References}

Abu-Hilal MM, Al-Baili MA, Sartawi A, Abdel-Fattah F, \& Al-Qarayout AI (2011). Psychometric Properties of the Wechsler Abbreviated Scale of Intelligence (WASI) with an Arab Sample of School Students. Individual Differences Research 9, 219-230.

Ala-Korpela M (2007a). Critical evaluation of 1H NMR metabonomics of serum as a methodology for disease risk assessment and diagnostics. Clinical Chemistry and Laboratory Medicine 46, 27-42.

Ala-Korpela M (2007b). Potential role of body fluid $1 \mathrm{H}$ NMR metabolomics as a prognostic and diagnostic tool. Expert Review of Molecular Diagnostics 7, 761773.

Auro K, Joensuu A, Fischer K, Kettunen J, Salo P, Mattsson H, Niironen M, Kaprio J, Eriksson JG, Lehtimäki T, Raitakari O, Jula A, Tiitinen A, Jauhiainen M, Soininen P, Kangas AJ, Kähönen M, Havulinna AS, Ala-Korpela M, Salomaa V, Metspalu A, \& Perola M (2014). A metabolic view on menopause and ageing. Nature Communications 5, 4708.

Blakemore SJ, \& Choudhury S (2006). Development of the adolescent brain: Implications for executive function and social cognition. Journal of Child Psychology and Psychiatry and Allied Disciplines 47, 296-312.

Bos DJ, Oranje B, Veerhoek ES, Van Diepen RM, Weusten JMH, Demmelmair H, Koletzko B, de Sain-van der Velden MGM, Eilander A, Hoeksma M, \& Durston S (2015). Reduced Symptoms of Inattention after Dietary Omega-3 Fatty Acid Supplementation in Boys with and without Attention Deficit/Hyperactivity Disorder. Neuropsychopharmacology 40, 2298-2306. Boyd A, Golding J, Macleod J, Lawlor DA, Fraser A, Henderson J, Molloy L, Ness A, Ring S, \& Smith GD (2013). Cohort profile: The "Children of the 90s"The index offspring of the avon longitudinal study of parents and children. International Journal of Epidemiology 42, 111-127.

Bradford Hill A (1965). The Environment and Disease: Association or Causation? Proceedings of the Royal Society of Medicine 58, 295-300.

Carrión RE, McLaughlin D, Goldberg TE, Auther AM, Olsen RH, Olvet DM, Correll CU, \& Cornblatt BA (2013). Prediction of Functional Outcome in Individuals at Clinical High Risk for Psychosis. JAMA Psychiatry 70, 1133-1142.

Coe DP, Peterson T, Blair C, Schutten MC, \& Peddie H (2013). Physical Fitness, Academic Achievement, and Socioeconomic Status in School-Aged Youth. Journal of School Health 83, 500-507.

Duckworth AL, \& Seligman MEP (2005). Self-discipline outdoes IQ in predicting academic performance of adolescents. Psychological Science 16, 939944.

Fett AKJ, Viechtbauer W, Dominguez M de G, Penn DL, van Os J, \& Krabbendam L (2011). The relationship between neurocognition and social cognition with functional outcomes in schizophrenia: A meta-analysis.

Neuroscience and Biobehavioral Reviews 35, 573-588.

Fischer K, Kettunen J, Würtz P, Haller T, Havulinna AS, Kangas AJ, Soininen P, Esko T, Tammesoo ML, Mägi R, Smit S, Palotie A, Ripatti S, Salomaa V, AlaKorpela M, Perola M, \& Metspalu A (2014). Biomarker Profiling by Nuclear Magnetic Resonance Spectroscopy for the Prediction of All-Cause Mortality: An Observational Study of 17,345 Persons. . Public Library of Science PLoS Medicine 
11, e1001606.

Fusar-Poli P, Borgwardt S, Bechdolf A, Addington J, Riecher-Rössler A, Schultze-Lutter F, Keshavan MS, Wood S, Ruhrmann S, Seidman LJ, Valmaggia L, Cannon T, Velthorst E, de Haan L, Cornblatt B, Bonoldi I, Birchwood M, Mcglashan TH, Carpenter WT, McGorry PD, Klosterkötter J, McGuire P, \& Yung AR (2013). The psychosis at risk state: a comprehensive state-of-the-art review. JAMA Psychiatry 70, 107-120.

Golding J, Pembrey M, \& Jones R (2001). ALSPAC--the Avon Longitudinal Study of Parents and Children. I. Study methodology. Paediatric and perinatal epidemiology 15, 74-87.

He Y, Yu Z, Giegling I, Xie L, Hartmann AM, Prehn C, Adamski J, Kahn R, Li Y, Illig T, Wang-Sattler R, \& Rujescu D (2012). Schizophrenia shows a unique metabolomics signature in plasma. Transl Psychiatry 2, e149.

Heinimaa M, Salokangas RK, Ristkari T, Plathin M, Huttunen J, Ilonen T, Suomela T, Korkeila J, \& McGlashan TH (2003). PROD-screen - a screen for prodromal symptoms of psychosis. Int J Methods Psychiatr Res 12, 92-104. Holmes E, Tsang TM, Huang JTJ, Leweke FM, Koethe D, Gerth CW, Nolden BM, Gross S, Schreiber D, Nicholson JK, \& Bahn S (2006). Metabolic profiling of CSF: Evidence that early intervention may impact on disease progression and outcome in schizophrenia. PLoS Medicine 3, 1420-1428.

Holshausen K, Bowie CR, Mausbach BT, Patterson TL, \& Harvey PD (2014). Neurocognition, functional capacity, and functional outcomes: The cost of inexperience. . Elsevier B.V. Schizophrenia Research 152, 430-434.

Hurtig TM, Taanila A, Veijola J, Ebeling H, Mäki P, Miettunen J, Kaakinen M, Joukamaa M, Therman S, Heinimaa M, Järvelin MR, \& Moilanen I (2011). Associations between psychotic-like symptoms and inattention/hyperactivity symptoms. Social Psychiatry and Psychiatric Epidemiology 46, 17-27.

Inouye M, Kettunen J, Soininen P, Silander K, Ripatti S, Kumpula LS, Hämäläinen E, Jousilahti P, Kangas AJ, Männistö S, Savolainen MJ, Jula A, Leiviskä J, Palotie A, Salomaa V, Perola M, Ala-Korpela M, \& Peltonen L (2010). Metabonomic, transcriptomic, and genomic variation of a population cohort. Molecular Systems Biology 6

Ishikawa M, Maekawa K, Saito K, Senoo Y, Urata M, Murayama M, Tajima Y, Kumagai Y, \& Saito Y (2014). Plasma and serum lipidomics of healthy white adults shows characteristic profiles by subjects' gender and age. PLOS ONE 9, 112.

Järvelin MR, Hartikainen-Sorri AL, \& Rantakallio P (1993). Labour induction policy in hospitals of different levels of specialisation. British journal of obstetrics and gynaecology 100, 310-315.

Kelleher I, Connor D, Clarke MC, Devlin N, Harley M, \& Cannon M (2012). Prevalence of psychotic symptoms in childhood and adolescence: a systematic review and meta-analysis of population-based studies. Psychological Medicine 42, 1857-63.

Kettunen J, Demirkan A, Würtz P, Draisma HHM, Haller T, Rawal R, Vaarhorst A, Kangas AJ, Lyytikäinen LP, Pirinen M, Pool R, Sarin AP, Soininen P, Tukiainen T, Wang Q, Tiainen M, Tynkkynen T, Amin N, Zeller T, Beekman M, Deelen J, Van Dijk KW, Esko T, Hottenga JJ, Van Leeuwen EM, Lehtimäki T, Mihailov E, Rose RJ, De Craen AJM, Gieger C, Kähönen M, Perola M, Blankenberg S, Savolainen MJ, Verhoeven A, Viikari J, Willemsen 
G, Boomsma DI, Van Duijn CM, Eriksson J, Jula A, Järvelin MR, Kaprio J, Metspalu A, Raitakari O, Salomaa V, Eline Slagboom P, Waldenberger M, Ripatti S, \& Ala-Korpela M (2016). Genome-wide study for circulating metabolites identifies 62 loci and reveals novel systemic effects of LPA. Nature Communications 7, 1-9.

Koivukangas J, Tammelin T, Kaakinen M, Mäki P, Moilanen I, Taanila A, \& Veijola J (2010). Physical activity and fitness in adolescents at risk for psychosis within the Northern Finland 1986 Birth Cohort. Schizophrenia Research 116, 152-158.

Koponen H, Maki P, Halonen H, Miettunen J, Laitinen J, Tammelin T, Moilanen I, Taanila A, Ruokonen A, Korkeila J, \& Veijola J (2008). Insulin resistance and lipid levels in adolescents with familial risk for psychosis. Acta psychiatrica Scandinavica 117, 337-341.

Kujala UM, Makinen VP, Heinonen I, Soininen P, Kangas AJ, Leskinen TH, Rahkila P, Wurtz P, Kovanen V, Cheng S, Sipila S, Hirvensalo M, Telama R, Tammelin T, Savolainen MJ, Pouta A, O'Reilly PF, Mantyselka P, Viikari J, Kahonen M, Lehtimaki T, Elliott P, Vanhala MJ, Raitakari OT, Jarvelin MR, Kaprio J, Kainulainen H, \& Ala-Korpela M (2013). Long-term leisure-time physical activity and serum metabolome. Circulation 127, 340-348.

Mahendran Y, Cederberg H, Vangipurapu J, Kangas AJ, Soininen P, Kuusisto J, Uusitupa M, Ala-Korpela M, \& Laakso M (2013). Glycerol and fatty acids in serum predict the development of hyperglycemia and type 2 diabetes in Finnish men. Diabetes Care 36, 3732-3738.

Mäki P, Koskela S, Murray GK, Nordström T, Miettunen J, Jääskeläinen E, \& Veijola JM (2014). Difficulty in making contact with others and social withdrawal as early signs of psychosis in adolescents-the Northern Finland Birth Cohort 1986. European Psychiatry 29, 345-351.

Mapstone M, Cheema AK, Fiandaca MS, Zhong X, Mhyre TR, MacArthur LH, Hall WJ, Fisher SG, Peterson DR, Haley JM, Nazar MD, Rich SA, Berlau DJ, Peltz CB, Tan MT, Kawas CH, \& Federoff HJ (2014). Plasma phospholipids identify antecedent memory impairment in older adults. Nature medicine $\mathbf{2 0}$, 415-8.

McIntyre RS, Cha DS, Soczynska JK, Woldeyohannes HO, Gallaugher LA, Kudlow P, Alsuwaidan M, \& Baskaran A (2013). Cognitive deficits and functional outcomes in major depressive disorder: Determinants, substrates, and treatment interventions. Depression and Anxiety 30, 515-527.

Metcalf SA, Jones PB, Nordstrom T, Timonen M, Mäki P, Miettunen J, Jääskeläinen E, Järvelin M-R, Stochl J, Murray GK, Veijola J, \& Khandaker GM (2017). Serum C-reactive protein in adolescence and risk of schizophrenia in adulthood: A prospective birth cohort study. Brain, Behavior, and Immunity 59, 253-259.

Miettunen J, Törmänen S, Murray GK, Jones PB, Mäki P, Ebeling H, Moilanen I, Taanila A, Heinimaa M, Joukamaa M, \& Veijola J (2008). Association of cannabis use with prodromal symptoms of psychosis in adolescence. The British journal of psychiatry : the journal of mental science 192, 470-471.

Miller KM, Price CC, Okun MS, Montijo H, \& Bowers D (2009). Is the N-back task a valid neuropsychological measure for assessing working memory? Archives of Clinical Neuropsychology 24, 711-717.

Murray RM, \& Lewis SW (1988). Is schizophrenia a neurodevelopmental 
disorder? British medical journal (Clinical research ed.) 296, 63.

Orešič M, Tang J, Seppänen-Laakso T, Mattila I, Saarni SI, Suoma E, Lönnqvist J, Sysi-Aho M, Hyötyläinen T, Perälä J, \& Suvisaari J (2011).

Metabolome in schizophrenia and other psychotic disorders: a general population-based study. Genome medicine 3, 19.

Paus T, Keshavan M, \& Giedd JN (2008). Why do many psychiatric disorders emerge during adolescence? Nature Reviews Neuroscience 9, 947-957.

Rapoport JL, Addington AM, Frangou S, \& Psych MRC (2005). The neurodevelopmental model of schizophrenia: update 2005. Molecular psychiatry 10, 434-449.

Reichenberg A, Caspi A, Harrington H, Houts R, Keefe RSE, Murray RM, Poulton R, \& Moffitt TE (2010). Static and dynamic cognitive deficits in childhood preceding adult schizophrenia: A 30-year study. American Journal of Psychiatry 167, 160-169.

Rohde TE, \& Thompson LA (2007). Predicting academic achievement with cognitive ability. Intelligence 35, 83-92.

Saklofske DH, Caravan G, \& Schwartz C (2000). Concurrent Validity of the Wechsler Abbreviated Scale of Intelligence (WASI) with a Sample of Canadian Children. Canadian Journal of School Psychology 16, 87-94.

Scoriels L, Salek RM, Goodby E, Grainger D, Dean AM, West JA, Griffin JL, Suckling J, Nathan PJ, Lennox BR, Murray GK, Bullmore ET, \& Jones PB (2015). Behavioural and molecular endophenotypes in psychotic disorders reveal heritable abnormalities in glutamatergic neurotransmission. Translational Psychiatry 5, e540.

Serbin LA, Zelkowitz P, Doyle AB, Gold D, \& Wheaton B (1990). The socialization of sex-differentiated skills and academic performance: A mediational model. Sex Roles 23, 613-628.

Soininen P, Kangas AJ, Würtz P, Suna T, \& Ala-Korpela M (2015). Quantitative serum nuclear magnetic resonance metabolomics in cardiovascular epidemiology and genetics. Circulation: Cardiovascular Genetics 8, 192-206.

Soininen P, Kangas AJ, Würtz P, Tukiainen T, Tynkkynen T, Laatikainen R, Järvelin M-R, Kähönen M, Lehtimäki T, Viikari J, Raitakari OT, Savolainen MJ, \& Ala-Korpela M (2009). High-throughput serum NMR metabonomics for cost-effective holistic studies on systemic metabolism. Analyst 134, 1781-1785.

Song L, Singh J, \& Singer M (1994). The Youth Self-Report inventory: A study of its measurements fidelity. Psychological Assessment 6, 236-245.

Taanila AM, Hurtig TM, Miettunen J, Ebeling HE, \& Moilonen IK (2009). Association between ADHD symptoms and adolescents' psychosocial well-being: a study of the Northern Finland Birth Cohort 1986. International Journal of Circumpolar Health 68, 133-144.

Therman S, Heinimaa M, Miettunen J, Joukamaa M, Moilanen I, Mäki P, \& Veijola J (2011). Symptoms associated with psychosis risk in an adolescent birth cohort: Improving questionnaire utility with a multidimensional approach. Early Intervention in Psychiatry 5, 343-348.

Trushina E, Dutta T, Persson XMT, Mielke MM, \& Petersen RC (2013). Identification of Altered Metabolic Pathways in Plasma and CSF in Mild Cognitive Impairment and Alzheimer's Disease Using Metabolomics. PLoS ONE 8, e63644. Tukiainen T, Tynkkynen T, Mäkinen V-P, Jylänki P, \& Kangas A (2008). Biochemical and Biophysical Research Communications A multi-metabolite 
analysis of serum by 1 H NMR spectroscopy : Early systemic signs of Alzheimer's disease. 375, 356-361.

University of Oulu (2017). Northern Finland Cohorts

Wang Q, Kangas AJ, Soininen P, Tiainen M, Tynkkynen T, Puukka K, Ruokonen A, Viikari J, Kähönen M, Lehtimäki T, Salomaa V, Perola M, Smith GD, Raitakari OT, Järvelin MR, Würtz P, Kettunen J, \& Ala-Korpela M (2015). Sex hormone-binding globulin associations with circulating lipids and metabolites and the risk for type 2 diabetes: Observational and causal effect estimates. International Journal of Epidemiology 44, 623-637.

Würtz P, Cook S, Wang Q, Tiainen M, \& Tynkkynen T (2016). Metabolic profiling of alcohol consumption in 9778 young adults. International Journal of Epidemiology 45, 1493-1506.

Würtz P, Havulinna AS, Soininen P, Tynkkynen T, Prieto-Merino D, Tillin T, Ghorbani A, Artati A, Wang Q, Tiainen M, Kangas AJ, Kettunen J, Kaikkonen J, Mikkilä V, Jula A, Kähönen M, Lehtimäki T, Lawlor DA, Gaunt TR, Hughes AD, Sattar N, Illig T, Adamski J, Wang TJ, Perola M, Ripatti S, Vasan RS, Raitakari OT, Gerszten RE, Casas JP, Chaturvedi N, Ala-Korpela M, \& Salomaa V (2015). Metabolite profiling and cardiovascular event risk: A prospective study of 3 population-based cohorts. . Lippincott Williams and Wilkins Circulation 131, 774-785.

Würtz P, Mäkinen VP, Soininen P, Kangas AJ, Tukiainen T, Kettunen J, Savolainen MJ, Tammelin T, Viikari JS, Rönnemaa T, Kähönen M, Lehtimäki T, Ripatti S, Raitakari OT, Järvelin MR, \& Ala-Korpela M (2012). Metabolic signatures of insulin resistance in 7,098 young adults. Diabetes 61, 1372-1380. Würtz P, Wang Q, Kangas AJ, Richmond RC, Skarp J, Tiainen M, Tynkkynen T, Soininen P, Havulinna AS, Kaakinen M, Viikari JS, Savolainen MJ, Kähönen M, Lehtimäki T, Männistö S, Blankenberg S, Zeller T, Laitinen J, Pouta A, Mäntyselkä P, Vanhala M, Elliott P, Pietiläinen KH, Ripatti S, Salomaa V, Raitakari OT, Järvelin MR, Smith GD, \& Ala-Korpela M (2014). Metabolic Signatures of Adiposity in Young Adults: Mendelian Randomization Analysis and Effects of Weight Change. PLoS Medicine 11, e1001765.

Yang J, Chen T, Sun L, Zhao Z, Qi X, Zhou K, Cao Y, Wang X, Qiu Y, Su M, Zhao A, Wang P, Yang P, Wu J, Feng G, He L, Jia W, \& Wan C (2013). Potential metabolite markers of schizophrenia. Molecular psychiatry 18, 67-78.

Zammit S, Odd D, Horwood J, Thompson A, Thomas K, Menezes P, Gunnell D, Hollis C, Wolke D, Lewis G, \& Harrison G (2009). Investigating whether adverse prenatal and perinatal events are associated with non-clinical psychotic symptoms at age 12 years in the ALSPAC birth cohort. Psychological medicine 39, 1457-1467. 
Table 1. Characteristics of the two cohorts

\begin{tabular}{|c|c|c|c|c|}
\hline \multirow[t]{2}{*}{ Variable } & \multicolumn{2}{|l|}{ NFBC 1986} & \multicolumn{2}{|l|}{ ALSPAC } \\
\hline & $\begin{array}{l}\text { Higher academic } \\
\text { score }\end{array}$ & $\begin{array}{l}\text { Lower academic } \\
\text { score }\end{array}$ & $\begin{array}{l}\text { Higher academic } \\
\text { score }\end{array}$ & $\begin{array}{l}\text { Lower academic } \\
\text { score }\end{array}$ \\
\hline \multicolumn{5}{|l|}{ Demographic status } \\
\hline \multicolumn{5}{|l|}{ Sex } \\
\hline Male & $3,536(76 \%)$ & $1,110(24 \%)$ & $4,886(81 \%)$ & $1,144(19 \%)$ \\
\hline Female & $3,988(90 \%)$ & $428(10 \%)$ & $5,233(88 \%)$ & $713(12 \%)$ \\
\hline \multicolumn{5}{|l|}{ Maternal education/social class } \\
\hline $\begin{array}{l}\text { Least/classes III- } \\
M / I V / V\end{array}$ & $1,537(75 \%)$ & $516(25 \%)$ & $1,612(81 \%)$ & $388(19 \%)$ \\
\hline Middle/IV-NM & $2,886(82 \%)$ & $647(18 \%)$ & $3,506(89 \%)$ & $448(11 \%)$ \\
\hline Most/class I, II & $2,123(93 \%)$ & $151(7 \%)$ & $2,488(93 \%)$ & $179(7 \%)$ \\
\hline \multicolumn{5}{|l|}{ Mental health status } \\
\hline \multicolumn{5}{|l|}{ Psychosis risk } \\
\hline Lower risk & $5,171(87 \%)$ & $785(13 \%)$ & $3,677(95 \%)$ & $184(5 \%)$ \\
\hline Higher risk & $343(86 \%)$ & $57(14 \%)$ & $175(86 \%)$ & $28(14 \%)$ \\
\hline Depression and anxiety scores & $20.127(4.347)$ & $19.463(4.310)$ & - & - \\
\hline \multicolumn{5}{|l|}{$\begin{array}{l}\text { Taking medication for anxiety or } \\
\text { depression }\end{array}$} \\
\hline No & $6,073(86 \%)$ & $969(14 \%)$ & - & - \\
\hline Yes & $46(84 \%)$ & $9(16 \%)$ & - & - \\
\hline \multicolumn{5}{|l|}{ Physical health status } \\
\hline Body mass index - mean (SD) & $21.1(3.4)$ & $21.4(4.0)$ & $22.8(4.1)$ & $24.2(5.7)$ \\
\hline \multicolumn{5}{|l|}{ Visits to doctor in past 6 months } \\
\hline $0-1$ visits & $4,216(87 \%)$ & $630(13 \%)$ & & \\
\hline $2+$ visits & $1,649(86 \%)$ & $275(14 \%)$ & & \\
\hline \multicolumn{5}{|l|}{ Taking insulin } \\
\hline No & $5,945(86 \%)$ & $931(14 \%)$ & & \\
\hline Yes & $24(92 \%)$ & $2(8 \%)$ & & \\
\hline \multicolumn{5}{|l|}{ Physical activity } \\
\hline$>1$ hour/week & $3,913(89 \%)$ & $501(11 \%)$ & & \\
\hline$\leq 1$ hour/week & $2,020(82 \%)$ & $433(18 \%)$ & & \\
\hline \multicolumn{5}{|l|}{ Diet } \\
\hline Healthy & $3,861(91 \%)$ & $375(41 \%)$ & & \\
\hline Unhealthy & $2,068(79 \%)$ & $543(21 \%)$ & & \\
\hline
\end{tabular}


Table 5: Cross-sectional associations between metabolic measures and academic performance at age 16 years, controlling for mental health factors and physical health factors

\begin{tabular}{|c|c|c|c|c|c|}
\hline Variable & $\begin{array}{l}\text { Model } 3 \\
\text { OR }(95 \% \mathrm{Cl})\end{array}$ & $\begin{array}{l}\text { Model } 4 \text { (Model } \\
3+\text { anxiety and } \\
\text { depression) } \\
\text { OR }(95 \% \mathrm{Cl})\end{array}$ & $\begin{array}{l}\text { Model } 5 \text { (Model } \\
4^{\wedge}+\text { psychosis } \\
\text { risk) } \\
\text { OR }(95 \% \mathrm{Cl})\end{array}$ & $\begin{array}{l}\mathrm{P} \text { for interaction } \\
\text { (model } 5 \text { vs. } \\
\text { model with } \\
\text { interaction) }\end{array}$ & $\begin{array}{l}\text { Model } 6 \text { (Model } \\
3+\text { doctor visits, } \\
\text { exercise and } \\
\text { diet) OR }(95 \% \\
\mathrm{Cl})\end{array}$ \\
\hline \multicolumn{6}{|l|}{ NFBC sample } \\
\hline LDL diameter & $0.87(0.80,0.95)$ & $0.83(0.75,0.92)$ & $0.81(0.73,0.90)$ & 0.120 & $0.85(0.77,0.94)$ \\
\hline MUFA/total FA & $1.20(1.10,1.31)$ & $1.22(1.11,1.35)$ & $1.22(1.11,1.35)$ & 0.502 & $1.23(1.11,1.36)$ \\
\hline Omega-3/total FA & $0.80(0.73,0.88)$ & $0.82(0.74,0.91)$ & $0.83(0.74,0.93)$ & 0.683 & $0.81(0.73,0.91)$ \\
\hline DHA/total FA & $0.86(0.79,0.95)$ & $0.88(0.79,0.97)$ & $0.89(0.80,0.99)$ & 0.918 & $0.87(0.78,0.96)$ \\
\hline \multicolumn{6}{|l|}{ ALSPAC sample } \\
\hline LDL diameter & $0.78(0.68,0.91)$ & NA & $0.80(0.69,0.93)$ & 0.417 & NA \\
\hline MUFA/total FA & $1.29(1.13,1.48)$ & NA & $1.26(1.10,1.45)$ & 0.909 & NA \\
\hline Omega-3/total FA & $0.65(0.55,0.76)$ & NA & $0.66(0.56,0.78)$ & 0.300 & NA \\
\hline DHA/total FA & $0.67(0.57,0.78)$ & NA & $0.67(0.57,0.79)$ & 0.496 & NA \\
\hline \multicolumn{6}{|c|}{ Model 3 controlling for sex, maternal education level and body mass index } \\
\hline \multicolumn{6}{|c|}{${ }^{\wedge}$ Model 3 in the case of the ALSPAC sample } \\
\hline \multicolumn{6}{|c|}{ OR=odds ratio; 95\% Cl=95\% confidence interval; LDL=Low Density Lipoprotein; HDL=High Density Lipoprotein; } \\
\hline \multicolumn{6}{|c|}{ MUFA=Monounsaturated Fatty Acids; FA=Fatty Acids; DHA=Docosahexaenoic Acid } \\
\hline
\end{tabular}

Fig. 1. Overview of cross-sectional and longitudinal measurements

Fig. 2. Cross-sectional associations between metabolic measures and cognitive performance in adolescence, controlling for sex

Fig. 3. Longitudinal associations between metabolic measures and cognitive performance in adolescence, controlling for sex

Supplementary Fig. 1. Cross-sectional associations between metabolic measures and executive function at age 17 years, controlling for sex

Supplementary Fig. 2. Cross-sectional associations between metabolic measures and psychosis risk in adolescence, controlling for sex

Supplementary Fig. 3. Longitudinal associations between metabolic measures at 15 years and executive function and psychosis risk at 17 years, controlling for sex 
Table 2. Cross-sectional associations between metabolic measures and cognitive performance, controlling for covariates

\begin{tabular}{|c|c|c|c|c|c|c|}
\hline \multirow[t]{2}{*}{ Metabolic measure } & \multicolumn{3}{|c|}{ NFBC $1986(n=5,510)$} & \multicolumn{3}{|c|}{ ALSPAC $(n=3,022)$} \\
\hline & Model 1 & Model 2 & Model 3 & Model 1 & Model 2 & Model 3 \\
\hline Lipids in small VLDL & $1.13(1.04,1.22)$ & $1.08(0.99,1.18)$ & $1.08(0.99,1.18)$ & - & - & - \\
\hline Lipids in very large HDL & $0.88(0.81,0.95)$ & $0.92(0.84,1.00)$ & $0.92(0.84,1.01)$ & - & - & - \\
\hline Lipids in small HDL & - & - & - & $1.21(1.10,1.33)$ & $1.27(1.14,1.42)$ & $1.32(1.16,1.50)$ \\
\hline LDL diameter & $0.85(0.79,0.93)$ & $0.87(0.80,0.95)$ & $0.87(0.80,0.95)$ & $0.85(0.076,0.95)$ & $0.83(0.74,0.94)$ & $0.78(0.68,0.91)$ \\
\hline HDL diameter & $0.84(0.78,0.91)$ & $0.89(0.81,0.97)$ & $0.89(0.81,0.97)$ & $0.85(0.76,0.94)$ & $0.87(0.77,0.98)$ & $0.95(0.82,1.09)$ \\
\hline Serum TG & $1.14(1.05,1.23)$ & $1.08(1.00,1.18)$ & $1.08(0.99,1.18)$ & - & - & - \\
\hline TG in VLDL & $1.12(1.04,1.22)$ & $1.07(0.99,1.17)$ & $1.07(0.98,1.17)$ & - & - & - \\
\hline TG in LDL & $1.15(1.06,1.25)$ & $1.12(1.02,1.22)$ & $1.12(1.02,1.22)$ & - & - & - \\
\hline PL in VLDL & $1.12(1.04,1.21)$ & $1.07(0.99,1.17)$ & $1.07(0.98,1.17)$ & - & - & - \\
\hline MUFAs & $1.18(1.09,1.27)$ & $1.15(1.05,1.25)$ & $1.15(1.05,1.25)$ & - & - & - \\
\hline Omega-3 FAs & - & - & - & $0.78(0.70,0.87)$ & $0.82(0.73,0.94)$ & $0.76(0.65,0.88)$ \\
\hline $\mathrm{DHA}$ & - & - & - & $0.71(0.63,0.80)$ & $0.76(0.66,0.87)$ & $0.74(0.63,0.87)$ \\
\hline MUFA/Total FA & $1.24(1.15,1.34)$ & $1.20(1.11,1.31)$ & $1.20(1.10,1.31)$ & $1.22(1.10,1.35)$ & $1.23(1.10,1.38)$ & $1.29(1.13,1.48)$ \\
\hline PUFA/Total FA & $0.80(0.74,0.86)$ & $0.83(0.76,0.90)$ & $0.83(0.76,0.90)$ & - & - & - \\
\hline Omega-6/Total FA & $0.83(0.77,0.90)$ & $0.87(0.80 .0 .94)$ & $0.87(0.80,0.94)$ & - & - & - \\
\hline LA/Total FA & $0.86(0.80,0.93)$ & $0.89(0.82,0.97)$ & $0.89(0.82,0.97)$ & - & - & - \\
\hline Omega-3/Total FA & $0.80(0.73,0.87)$ & $0.80(0.73,0.88)$ & $0.80(0.73,0.88)$ & $0.71(0.64,0.80)$ & $0.74(0.65,0.84)$ & $0.65(0.55,0.76)$ \\
\hline DHA/Total FA & $0.85(0.79,0.93)$ & $0.87(0.79,0.95)$ & $0.86(0.79,0.94)$ & $0.65(0.58,0.74)$ & $0.69(0.61,0.79)$ & $0.67(0.57,0.78)$ \\
\hline Unsaturated FA \% & $0.79(0.73,0.86)$ & $0.82(0.75,0.89)$ & $0.81(0.75,0.89)$ & $0.85(0.77,0.94)$ & $0.86(0.77,0.96)$ & $0.91(0.80,1.04)$ \\
\hline Citrate & $0.82(0.76,0.89)$ & $0.84(0.77,0.92)$ & $0.84(0.77,0.92)$ & - & - & - \\
\hline Creatinine & $0.87(0.80,0.95)$ & $0.88(0.81,0.96)$ & $0.88(0.80,0.96)$ & - & - & - \\
\hline
\end{tabular}

Model 1 controlled for sex; Model 2 controlled for sex and maternal education/socioeconomic status; Model 3 controlled for sex, maternal education/socioeconomic status and body mass index VLDL=Very Low Density Lipoprotein; HDL=High Density Lipoprotein; LDL=Low Density Lipoprotein; TG=Triglycerides; PL=Phospholipids; FA=Fatty Acids; MUFA=Monounsaturated Fatty Acids; PUFA=Polyunsaturated Fatty Acids; DHA=Docosahexaenoic Acid Bold $=P<0.004$ 
Table 3. Longitudinal associations between metabolic measures and cognitive performance, controlling for covariates

\begin{tabular}{|c|c|c|c|c|c|c|}
\hline \multirow[t]{2}{*}{ Metabolic measure } & \multicolumn{3}{|c|}{ Academic scores $(n=2,893)$} & \multicolumn{3}{|c|}{ PR latency $(n=2,040)$} \\
\hline & Model 1 & Model 2 & Model 3 & Model 1 & Model 2 & Model 3 \\
\hline Lipids in large HDL & $0.75(0.64,0.89)$ & $0.86(0.70,1.05)$ & $0.77(0.58,1.04)$ & - & - & - \\
\hline LDL diameter & $0.80(0.68,0.93)$ & $0.70(0.59,0.84)$ & $0.62(0.48,0.80)$ & - & - & - \\
\hline HDL diameter & $0.76(0.64,0.89)$ & $0.83(0.68,1.01)$ & $0.74(0.56,0.98)$ & - & - & - \\
\hline MUFA & $1.23(1.07,1.41)$ & $1.36(1.16,1.60)$ & $1.47(1.18,1.83)$ & - & - & - \\
\hline PUFA & - & - & - & $0.79(0.69,0.91)$ & $0.75(0.64,0.88)$ & $0.74(0.63,0.87)$ \\
\hline Omega-6 & - & - & - & $0.79(0.69,0.91)$ & $0.77(0.65,0.90)$ & $0.76(0.65,0.89)$ \\
\hline Linoleic Acid & - & - & - & $0.81(0.70,0.93)$ & $0.79(0.68,0.92)$ & $0.79(0.67,0.92)$ \\
\hline Omega-3 & - & - & - & $0.80(0.70,0.92)$ & $0.75(0.64,0.89)$ & $0.74(0.63,0.87)$ \\
\hline $\mathrm{DHA}$ & $0.76(0.63,0.92)$ & $0.91(0.73,1.13)$ & $0.91(0.68,1.21)$ & $0.71(0.63,0.80)$ & $0.72(0.61,0.85)$ & $0.72(0.61,0.85)$ \\
\hline MUFA/Total FA & $1.35(1.16,1.56)$ & $1.32(1.11,1.58)$ & $1.50(1.18,1.90)$ & - & - & - \\
\hline PUFA/Total FA & $0.79(0.68,0.91)$ & $0.77(0.65,0.92)$ & $0.71(0.56,0.90)$ & - & - & - \\
\hline Omega-3/Total FA & $0.75(0.63,0.89)$ & 0.84 (0.68. 1.03) & $0.76(0.57,1.01)$ & - & - & - \\
\hline $\mathrm{DHA} /$ Total FA & $0.70(0.58,0.84)$ & $0.74(0.59,0.93)$ & $0.75(0.56,1.01)$ & - & - & - \\
\hline
\end{tabular}

Model 1 controlled for sex; Model 2 controlled for sex and maternal education/socioeconomic status; Model 3 controlled for sex, maternal education/socioeconomic status and body mass index

HDL=High Density Lipoprotein; LDL=Low Density Lipoprotein; FA=Fatty Acids; MUFA=Monounsaturated Fatty Acids; PUFA=Polyunsaturated Fatty Acids; DHA=Docosahexaenoic Acid

Bold $=\mathrm{P}<0.004$ 
Table 4. Cross-sectional associations between metabolic measures and various markers of brain function from age 15-17 years and longitudinal associations between metabolic measures at age 15 years and markers of brain function at age 16-17 in ALSPAC only

\begin{tabular}{|c|c|c|c|c|c|c|c|c|c|c|}
\hline \multirow[b]{2}{*}{ Variable } & \multicolumn{6}{|c|}{ Cross-sectional } & \multicolumn{4}{|c|}{ Longitudinal } \\
\hline & $\begin{array}{l}\text { ALSPAC } \\
\text { WASI (15) }\end{array}$ & $\begin{array}{l}\text { NFBC } \\
\text { academic (16) }\end{array}$ & $\begin{array}{l}\text { NFBC psychosis } \\
\text { risk (16) }\end{array}$ & $\begin{array}{l}\text { ALSPAC N- } \\
\text { back (17) }\end{array}$ & $\begin{array}{l}\text { ALSPAC PR } \\
\text { (17) }\end{array}$ & $\begin{array}{l}\text { ALSPAC } \\
\text { psychosis risk (17) }\end{array}$ & $\begin{array}{l}\text { Academic } \\
\text { (16) }\end{array}$ & $\begin{array}{l}\text { N-back } \\
(17)\end{array}$ & PR (17) & $\begin{array}{l}\text { Psychosis } \\
\text { risk (17) }\end{array}$ \\
\hline \multicolumn{11}{|l|}{ Lipoprotein subclass total lipids } \\
\hline Lipids in small HDL & $1.32^{* *}$ & $1.10^{*}$ & 1.04 & 0.96 & 1.01 & 0.82 & $1.34^{*}$ & 0.99 & 0.92 & 1.03 \\
\hline \multicolumn{11}{|l|}{ Lipoprotein particle size } \\
\hline LDL diameter & $0.78^{* *}$ & $0.87^{* *}$ & 0.99 & 1.01 & 1.05 & 1.11 & $0.62^{* \star}$ & 0.95 & $1.21^{*}$ & 0.98 \\
\hline \multicolumn{11}{|l|}{ Fatty acids } \\
\hline MUFA & $1.17^{*}$ & $1.15^{* *}$ & $1.09^{*}$ & 0.92 & 0.97 & 0.93 & $1.47^{* *}$ & 0.95 & 0.88 & 1.07 \\
\hline PUFA & 0.98 & 1.00 & $1.07^{*}$ & 0.94 & 0.91 & 0.99 & 1.04 & 1.07 & $0.74^{\star *}$ & 1.04 \\
\hline Omega-6 & 1.03 & 1.02 & $1.07^{*}$ & 0.96 & 0.93 & 1.00 & 1.06 & 1.10 & $0.76^{\star *}$ & 1.05 \\
\hline Linoleic acid & 1.02 & 1.03 & $1.07^{*}$ & 0.99 & 0.92 & 0.98 & 1.03 & 1.10 & $0.79^{* *}$ & 1.04 \\
\hline Omega-3 & $0.76^{\star *}$ & $0.91^{*}$ & $1.07^{*}$ & $0.82^{*}$ & $0.86^{*}$ & 0.94 & 0.95 & 0.91 & $0.74^{\star *}$ & 0.95 \\
\hline DHA & $0.74^{\star *}$ & 0.94 & $1.07^{*}$ & $0.77^{\star *}$ & 0.90 & 0.81 & 0.91 & 0.90 & $0.72^{\star *}$ & 0.93 \\
\hline \multicolumn{11}{|l|}{ Fatty acid ratios } \\
\hline MUFA & $1.29^{\star \star}$ & $1.20^{\star \star}$ & 1.06 & 0.94 & 1.04 & 0.93 & $1.50^{* *}$ & 0.93 & 1.14 & 1.06 \\
\hline PUFA & 0.90 & $0.83^{* *}$ & 0.97 & 1.11 & 0.97 & 1.08 & $0.71^{*}$ & $1.23^{*}$ & 0.96 & 0.95 \\
\hline Omega-3 & $0.65^{\star *}$ & $0.80^{* *}$ & 1.02 & 0.83 & $0.84^{*}$ & 0.94 & 0.76 & 0.90 & $0.85^{*}$ & 0.90 \\
\hline DHA & $0.67^{* *}$ & $0.86^{* *}$ & 1.03 & 0.76 & 0.89 & $0.78^{*}$ & 0.75 & 0.88 & $0.79^{* *}$ & 0.88 \\
\hline
\end{tabular}

HDL=High Density Lipoprotein; LDL=Low Density Lipoprotein; MUFA=Monounsaturated Fatty Acids; PUFA=Polyunsaturated Fatty Acids; DHA= Docosahexaenoic Acid

${ }^{* *} \mathrm{P}<0.004 ;{ }^{*} 0.004<\mathrm{P}<0.05$

NFBC 1986=Northern Finland Birth Cohort 1986; ALSPAC=Avon Longitudinal Study of Parents and Children 